\title{
A current-stepping technique to enhance pinch compression
}

\author{
S Lee \\ Plasma Research Laboratory, Physics Department, University of Malaya, \\ Kuala Lumpur 22-11, Malaysia
}

Received 22 September 1983

\begin{abstract}
It has been shown that the quasi-equilibrium radius ratio $K_{\mathrm{p}}$ of a fast compressional pinch is independent of the absolute magnitude of the driving current; it depends only on the current shape and on the length variation of the pinch during pinching. In this paper it is shown that a current step, switched on to the discharge current of a pinch as it approaches its quasi-equilibrium radius ratio $\kappa_{p}$ as defined by a lossless energy balance theory, shifts the value of $\kappa_{\mathrm{p}}$ to a significantly lower value, thus enhancing pinch compression. It is proposed that this current-stepping technique may be used as a prelude to radiation cooling to eventually achieve the very small radius ratios required for a pinch fusion reactor.
\end{abstract}

\section{Introduction}

A pinch fusion reactor concept has been proposed that fulfils the Lawson criterion and at the same time has enhanced stability due to large ion Larmor radius effects (Haines 1982). One of the key technical features of this proposal is the requirement of a small final pinch radius of the order of $10 \mu \mathrm{m}-100 \mu \mathrm{m}$. From the view point of fast compressional pinches the achievement of this order of pinch radius is a major difficulty as, even with the advent of new high-power electrical discharge technology, an initial radius of the order of $1 \mathrm{~cm}$ has to be used in order to provide sufficient running time of the pinch compression for effective energy transfer.

Thus a pinch radius ratio, that is the final radius divided by the initial radius, of 0.01 to 0.001 is required. Potter (1978), using a slug model, has shown that for a constant current pinch in deuterium the pinch ratio is fixed at 0.3 . Lee (1981) has proposed a lossless energy balance theory and used it to show that the pinch ratio is reduced to 0.14 for a plasma focus treated as a pinch with a length which increases as the radius decreases (Lee 1983a). The theory has also been used to show that for a constant length deuterium pinch, the radius ratio depends only on the current shape and varies from 0.17 for a linearly rising current (Lee 1983b) to 0.4 for an energy-optimum plasma-coupled $L-C$ discharge (Lee 1983d). The effect of specific heat ratio, $\gamma$, on the pinch ratio for argon (Lee 1983c) has also been computed.

It has therefore been demonstrated that based on a lossless theory the pinch radius ratio does not depend on the absolute magnitude of the current, but only on the time variation of the current pulse. For the various types of current functions discussed above 
the pinch ratios are relatively large. Haines (1982) has suggested the small radius ratios required for the pinch fusion reactor may be attained by radiation cooling compression, by operating the pinch at the Pease-Braginskii limiting current.

It is proposed in this paper that as a prelude to radiation cooling compression a technique of current stepping may be used to reduce the pinch ratio, thus enhancing plasma compression. This technique relies on the fact that when an equilibrium pinch ratio is approached as defined by the energy balance condition it is possible to shift the equilibrium pinch ratio to a reduced value by rapidly increasing or stepping-up the driving current.

\section{Theory}

The energy-balance theory (Lee 1981, 1983a) applied to the fast compression radial pinch equates the work done by the magnetic piston to the plasma enthalpy. The resultant expression for temperature, $T$, is compared to the temperature obtained from the Bennett pressure balance to obtain the following condition governing the quasiequilibrium pinch radius ratio:

$$
I_{\mathrm{p}}^{2}=\frac{2(\gamma-1)}{\gamma l_{\mathrm{p}}} \int_{r_{\mathrm{p}}}^{r_{0}} I^{2} l \frac{\mathrm{d} r}{r}
$$

where $I_{\mathrm{p}}$ is the current flowing at the time the pinch reaches quasi-equilibrium at $r_{\mathrm{p}}$ and $l_{\mathrm{p}}$ is the length of the pinch at quasi-equilibrium. The integration is done from initial radius $r_{0}$ to final radius $r_{\mathrm{p}}$.

For a constant length pinch we may rewrite equation (1) in the following normalised form:

$$
\iota_{\mathrm{p}}^{2}=\frac{2(\gamma-1)}{\gamma} \int_{\kappa_{\mathrm{p}}}^{1} \frac{\iota^{2} \mathrm{~d} K}{K}
$$

where $\iota=I / I_{0}, K=r / r_{0}$ and $I_{0}=V_{1} /\left(L_{1} / C_{1}\right)^{1 / 2}$ is the characteristic peak current of the $L-C$ discharge circuit.

The implication of this condition governing the quasi-equilibrium pinch position may be understood in terms of a radial snow-plough model coupled to a $L-C$ discharge (Lee 1983d). The $K$ and $\iota$ curves as functions of normalised $\tau=t / t_{0}$ (where $t_{0}=$ $\left.\left(L_{1} / C_{1}\right)^{1 / 2}\right)$ have the general form as depicted in figure $1(a)$. From these curves may be
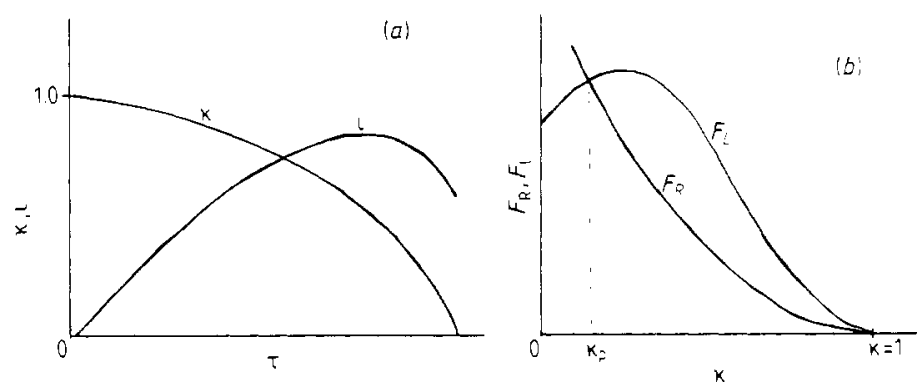

Figure 1. (a) General forms of snow-plough trajectory and circuit current as functions of time. (b) General forms of the functions $F_{L}$ and $F_{R}$ as derived from $(a)$. 
derived the curves of $F_{L}=\iota^{2}$ and

$$
F_{R}=\frac{2(\gamma-1)}{\gamma} \int_{\kappa}^{1} \frac{\iota^{2} \mathrm{~d} K}{K}
$$

as functions of $k$. The general forms of $F_{L}$ and $F_{R}$ are as depicted in figure $1(b)$. An analysis of $F_{L}$ and $F_{R}$ shows that at $K<1, F_{L}$ is larger than $F_{R}$ but that as $K \rightarrow 0$, the value of $F_{R}$ must exceed $F_{L}$ at some point. The point where the two curves intersect is the point at which condition (2) is met and the quasi-equilibrium radius ratio $\kappa_{\mathrm{p}}$ is achieved. It is not energetically possible to compress beyond this point.

As the compression proceeds $F_{R}$ rises to meet $F_{L}$ at a value of $K$ corresponding to $\kappa_{\mathrm{p}}$. If just before $\kappa_{\mathrm{p}}$ is reached the current $\iota$ is raised rapidly, or stepped up, the value of $F_{L}$ will increase faster than the value of $F_{R}$ because $F_{L}$ is the absolute value of the current squared whereas $F_{R}$ is an integral. Thus it is possible to diverge the values of $F_{L}$ and $F_{R}$ and move the quasi-equilibrium point to a lower value of $\kappa_{\mathrm{p}}$. A simple example with a single current step added to the primary current will now be discussed.

\section{The model}

Consider the circuit as shown in figure 2 . The switch $S_{1}$ in the primary circuit is closed so that the capacitor $C_{1}$ charged to voltage $V_{1}$ starts to discharge with current $I_{1}$ through inductance $L_{1}$ into the pinch of varying inductance $L_{\mathrm{p}}$. The pinch is depicted in coaxial form with pinching current flowing at radius $r$, and the return current in the return conductor of radius $r_{0}$. The initial radius of the pinch is approximated as $r_{0}$. As the pinch approaches its equilibrium position as determined by equation (2), the switch $S_{2}$ in the current-stepping circuit is closed so that the capacitor $C_{2}$ starts discharging current $I_{2}$ through inductance $L_{2}$ into the pinch.

Applying the snow-plough model to govern the pinch dynamics we have:

$$
\frac{\mathrm{d}}{\mathrm{d} t}\left(\pi \rho_{0}\left(r_{0}^{2}-r^{2}\right) \frac{\mathrm{d} r}{\mathrm{~d} t}\right)=-\frac{\mu I^{2}}{4 \pi r}
$$

where

$$
I=I_{1}+I_{2}
$$

The currents $I_{1}$ and $I_{2}$ are governed by the circuit equations:

$$
\frac{\mathrm{d}}{\mathrm{d} t}\left(L_{1} I_{1}\right)+\frac{\mathrm{d}}{\mathrm{d} t}\left(L_{\mathrm{p}} I\right)=V_{1}-\frac{\int I_{1} \mathrm{~d} t}{C_{1}}
$$

with

$$
I_{2}=0
$$

until switch $\mathrm{S}_{2}$ is closed; whereupon

$$
\frac{\mathrm{d}}{\mathrm{d} t}\left(L_{2} I_{2}\right)+\frac{\mathrm{d}}{\mathrm{d} t}\left(L_{\mathrm{p}} I\right)=V_{2}-\frac{\int I_{2} \mathrm{~d} t}{C_{2}}
$$

The plasma inductance $L_{\mathrm{p}}$ is given by

$$
L_{\mathrm{p}}=\frac{\mu}{2 \pi}\left(\ln \frac{r_{0}}{r}\right) l
$$




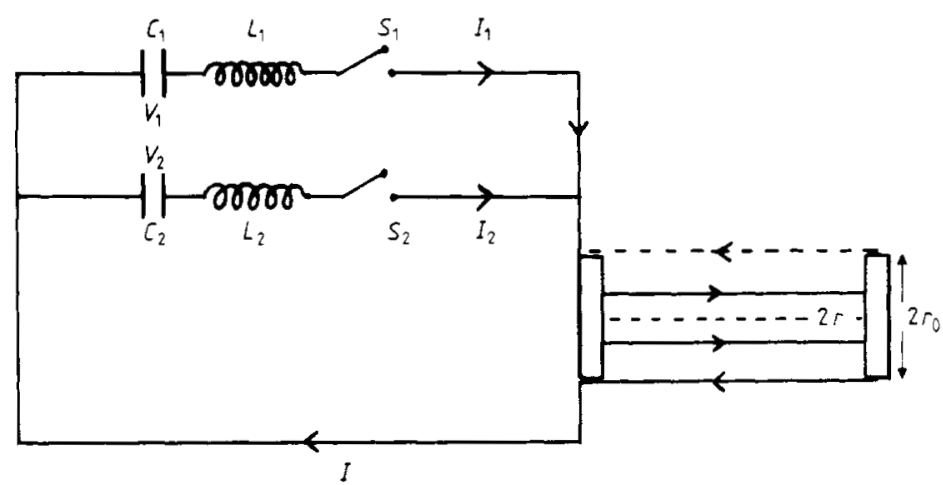

Figure 2. Schematic diagram of the arrangement for a current-stepping pinch.

The decision of when to switch on $\mathrm{S}_{2}$ may be related to how close the condition as expressed in equation (4) is approached. For the purpose of numerical computation we may define a convergence ratio as:

$$
\psi=\frac{2(\gamma-1)}{\gamma} \int_{\kappa}^{1} \frac{\iota^{2} \mathrm{~d} K}{\kappa} \iota^{-2}
$$

and numerically we may experiment with switching on $S_{2}$ at the time when $\psi$ attains a value such as $\psi_{0}=0.8$.

\section{Normalisation}

To formulate the problem in terms of relevant scaling parameters we adopt the following normalisation:

$$
\begin{array}{ll}
\tau=t / t_{0} & \kappa=r / r_{0} \\
\iota=I / I_{0} & \iota_{1}=I_{1} / I_{0} \quad \iota_{2}=I_{2} / I_{0} .
\end{array}
$$

Then the governing equations may be written in the form:

Motion:

$$
\frac{\mathrm{d}^{2} K}{\mathrm{~d} \tau^{2}}=\left[-\alpha_{0}^{2} \frac{\iota^{2}}{K}+2 \kappa\left(\frac{\mathrm{d} \kappa}{\mathrm{d} \tau}\right)^{2}\right]\left(1-\kappa^{2}\right)^{-1}
$$

Primary current circuit:

$$
\frac{\mathrm{d} \iota_{1}}{\mathrm{~d} \tau}=\left(1-\int \iota_{1} \mathrm{~d} \tau+\beta_{0} \frac{\iota}{\kappa} \frac{\mathrm{d} \kappa}{\mathrm{d} \tau}+\beta_{0} \ln \kappa \frac{\mathrm{d} \iota_{2}}{\mathrm{~d} \tau}\right)\left(1-\beta_{0} \ln \kappa\right)^{-1}
$$

Current-stepping circuit:

$$
\frac{\mathrm{d} \iota_{2}}{\mathrm{~d} \tau}=0
$$

or

$$
\frac{\mathrm{d} \iota_{2}}{\mathrm{~d} \tau}=\left(\frac{\lambda}{\beta}-\frac{1}{\beta \delta} \int \iota^{2} \mathrm{~d} \tau+\frac{\beta_{0}}{\beta} \frac{\iota}{K} \frac{\mathrm{d} \kappa}{\mathrm{d} \tau}+\frac{\beta_{0}}{\beta} \ln \kappa \frac{\mathrm{d} \iota_{1}}{\mathrm{~d} \tau}\right)\left(1-\frac{\beta_{0}}{\beta} \ln \kappa\right)^{-1}
$$


Total current:

$$
\iota=\iota_{1}+\iota_{2}
$$

and the switching condition for equation (12) is specified by presetting a value for $\psi$ in equation (9).

The three characteristic quantities in the set of equations are taken to be $r_{0}$, the initial radius of the pinch column, and $t_{0}$ and $I_{0}$ which are selected to relate to the primary current circuit:

$$
t_{0}=\left(L_{1} C_{1}\right)^{1 / 2} \quad I_{0}=V_{1} /\left(L_{1} / C_{1}\right)^{1 / 2}
$$

Then the scaling parameters which appear naturally in the equation are:

$$
\begin{aligned}
& \alpha_{0}=t_{0} / t_{\mathrm{p}} \\
& \beta_{0}=\frac{\mu l}{2 \pi} / L_{1} \\
& \beta=L_{2} / L_{1} \\
& \delta=C_{2} / C_{1} \\
& \lambda=V_{2} / V_{1}
\end{aligned}
$$

where

$$
t_{\mathrm{p}}=\left(\frac{4 \pi^{2} \rho_{0} r_{0}^{4}}{\mu I^{2}}\right)^{1 / 2}
$$

appears naturally as the characteristic time for the pinching process. The length of the pinch is here denoted as $l$.

Thus $\alpha_{0}$ is a scaling parameter for matching the characteristic discharge time of the primary current circuit to the characteristic pinching time of the system and $\beta_{0}$ is the scaling parameter for matching the characteristic pinch inductance to the primary circuit inductance $L_{1}$. The other scaling parameters $\beta, \delta, \lambda$ allow variation to be made in the ratio of the electrical parameters of the primary circuit to those of the current-stepping circuit. The preset ratio $\psi_{0}$ may also be considered as a parameter for selection of switching time for the current step.

\section{Computation procedure}

The scaling parameters $\alpha_{0}, \beta_{0}, \lambda, \delta, \beta$ and $\psi_{0}$ are set at desired values. The numerical computation is then initiated by setting $\tau=0, k=1, \mathrm{~d} K / \mathrm{d} \tau=0, \int \iota_{1} \mathrm{~d} \tau=0 . \iota_{1}=0$. $\mathrm{d} \iota_{1} / \mathrm{d} \tau=1$ are fixed initial conditions. The initial value of the acceleration $\mathrm{d}^{2} \kappa / \mathrm{d} \tau^{2}$ is not defined by equation (10), but it is found that a trial initial value of -1 is suitable for the numerical integeration to converge quickly. The other initial conditions are set as $\int \iota_{2} \mathrm{~d} \tau=0, \iota_{2}=0$ and $\mathrm{d} \iota_{2} / \mathrm{d} \tau=0$.

Equations (10), (11), (12a) and (13) are then integrated numerically by a linear approximation method which is found to give sufficient accuracy with a time step of 0.001 ; the results agree with integration by a Runge-Kutta scheme. At each step of the computation $\psi_{0}$ is computed numerically by equation (9) and compared with the preset value of $\psi$. When $\psi \geqslant \psi_{0}$ the equations for integration are changed to equations (10), 
(11), (12b) and (13). The integration continues until the value of $\psi$ attains $\psi>1$ at which point the energy balance condition as expressed by equation (4) has been reached. The integration is then stopped. The computation is performed for a $\gamma=5 / 3$ plasma.

\section{Results}

The results for two sets of parameters are presented here to demonstrate the capability of the technique to significantly reduce the radius ratio of the pinch. The first set of parameters, $\alpha_{0}=0.7, \beta_{0}=1, \beta=10, \delta=0.01, \lambda=30, \psi_{0}=0.8$, is suitable for a system where the primary circuit is a capacitor bank and the current step is provided by a second capacitor bank charged to 30 times the voltage of the primary bank. This might in practice be a capacitor bank-Marx combination. The values of $\alpha_{0}=0.7$ and $\beta_{0}=1$ ensure a good energy transfer between the primary bank and the plasma has occurred (Lee 1983d) at about the time the current step is switched. Under these conditions, the value of $\kappa_{\mathrm{p}}$ would be about 0.4 in the absence of the current step. The effect of the current step is quite significant as is seen in figure $3(b)$, which shows the values of $F_{L}$ and $F_{R}$ converging as $K$ approaches 0.4 and then diverging as the current is stepped, until a final convergence at $\kappa_{\mathrm{p}}=0.24$. Figure $3(a)$ shows the trajectory $\kappa$ versus $\tau$ and the current shape $\iota$ versus $\tau$.
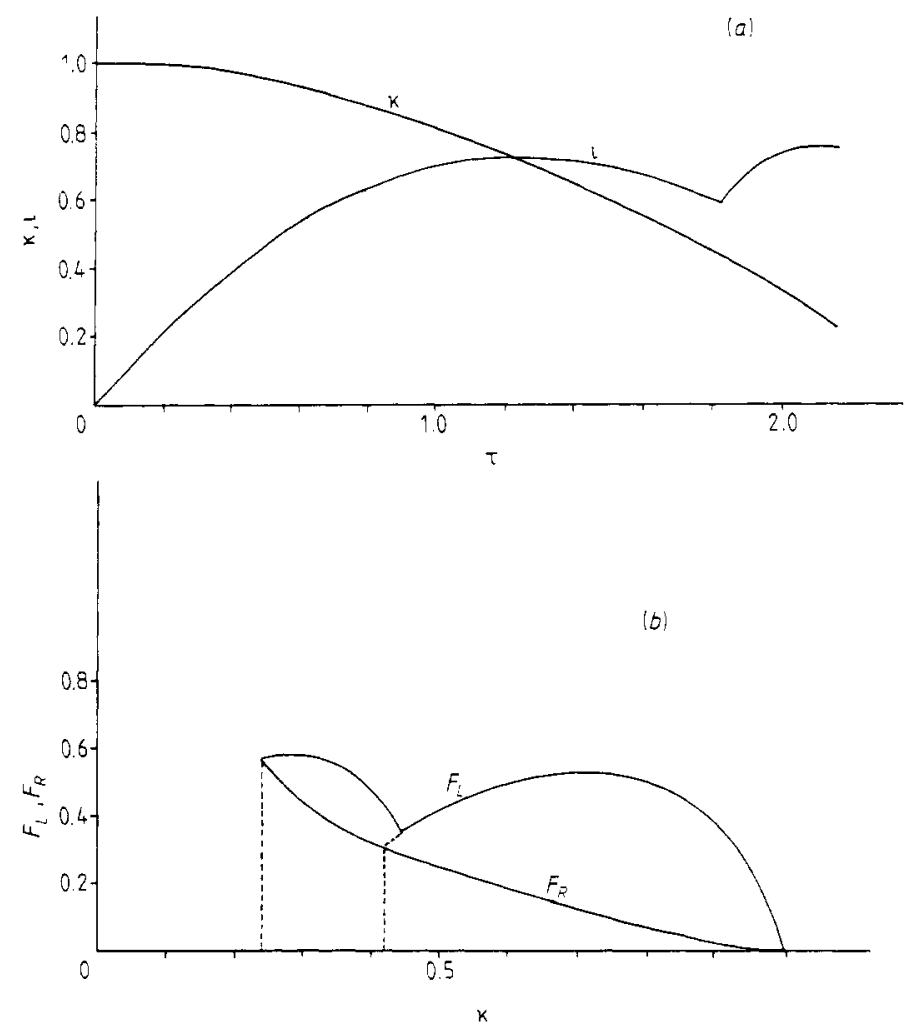

Figure 3. (a) Computed values of $\boldsymbol{k}$ and $\iota$ versus $\tau$ for $\alpha_{0}=0.7, \beta_{0}=1, \beta=10, \delta=0.01$, $\lambda=30, \psi_{0}=0.8$. (b) Computed values of $F_{L}$ and $F_{R}$ versus $k$ showing the shift in the energy balance point due to the current step for $\alpha_{0}=0.7, \beta_{0}=1, \beta=10, \delta=0.01, \lambda=30, \psi_{0}=$ 0.8 . 
The second set of parameters, $\alpha_{0}=1, \beta_{0}=0.1, \beta=0.25, \delta=2, \lambda=2, \psi_{0}=0.8$, may be more suitable for a system powered by two conventional capacitor banks, with the second bank having twice the voltage and capacitance as the first but only one quarter the inductance. With $\beta_{0}=0.1$, the energy efficiency of the first bank is relatively low (Lee 1983d). Under this condition the value of $\kappa_{\mathrm{p}}$ would be about 0.25 in the absence of a current step. Figure $4(b)$ shows the effect of switching on this current step. The quasi-equilibrium position is seen to have been shifted dramatically to $K_{\mathrm{p}}=0.14$. Incidentally this compression already equals that of the plasma focus which typically (Lee 1983a) has a $\kappa_{\mathrm{p}}$ also of 0.14 .
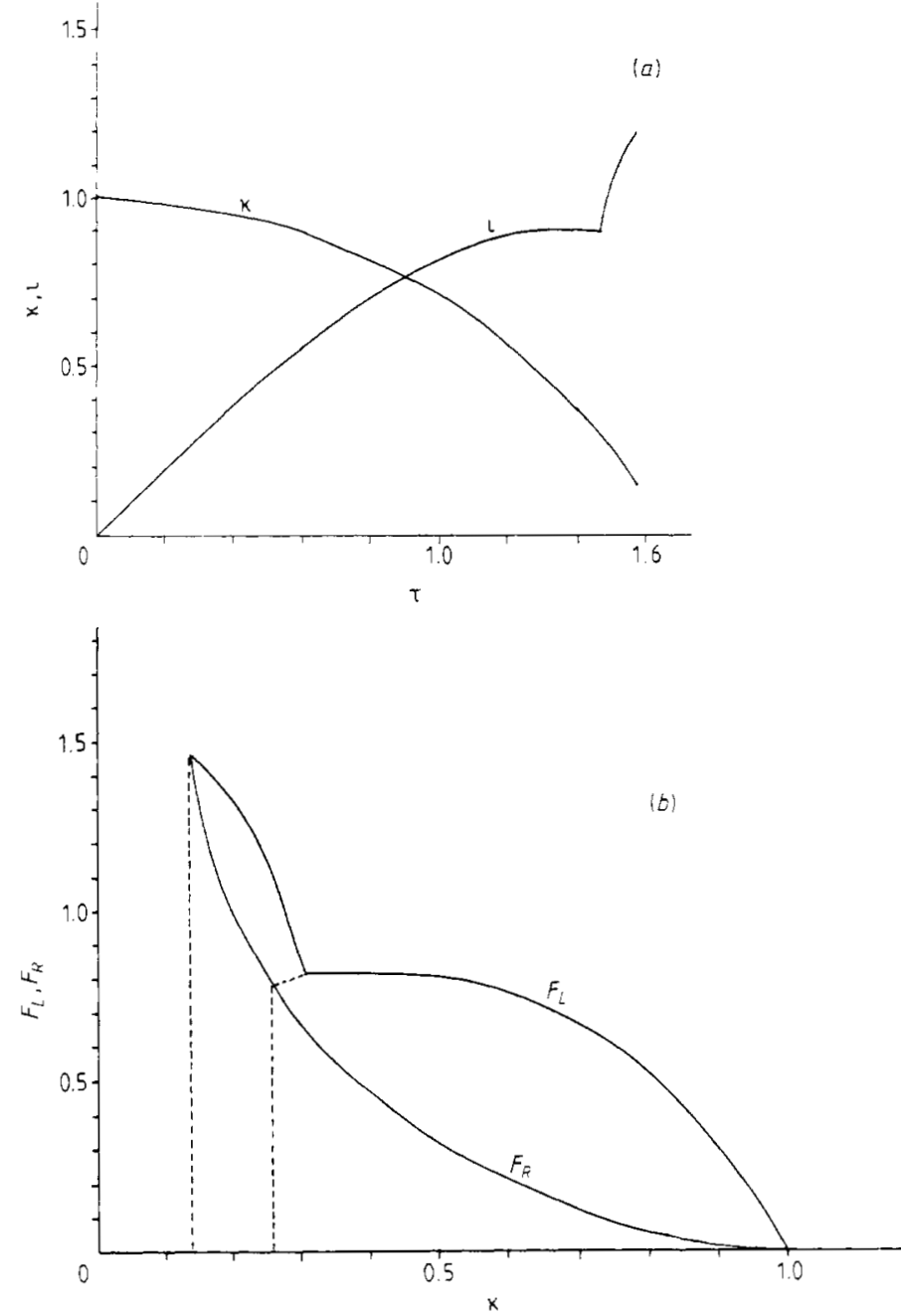

Figure 4. (a) Computed values of $k$ and $\iota$ versus $\tau$ for $\alpha_{0}=1, \beta_{0}=0.1, \beta=0.25, \delta=2, \lambda=$ 2, $\psi_{0}=0.8$. (b) Computed values of $F_{Z}$ and $F_{R}$ versus $x$ showing the shift in the energy balance point due to the current step for $\alpha_{0}=1, \beta_{0}=0.1, \beta=0.25, \delta=2, \lambda=2, \psi_{0}=0.8$.

For a practical situatior it is necessary to consider the possibility of excessively large voltage reversals across the first capacitor $C_{1}$. This is particularly the case in the first example considered since the second capacitor $C_{2}$, at 30 times the charging voltage of 
$C_{1}$, is switched on to both the pinch as well as $C_{1}$ at a time when the voltage across $C_{1}$ is near zero and its current is dropping from its peak value. It is found that the effect on the current $I_{1}$ is to force its value down faster than it would have fallen had $C_{2}$ not been switched. However because the value of $\left(L_{2} C_{2}\right)^{1 / 2}$ is only 0.32 times the value of $\left(L_{1} C_{1}\right)^{1 / 2}$, a consequence of the parameters $\beta=10, \delta=0.01$, the voltage on $C_{2}$ has dropped through zero soon after $I_{1}$ has been forced through zero.

In order to ensure that in each case the current or voltage on $C_{1}$ do not swing past acceptable limits in a discharge, the behaviour of the currents and voltages have been followed by continuing the integration of equations (11) and $(12 b)$ up to $\tau=6.3$, representing one complete cycle of the short-circuited $L_{1}-C_{1}$ combination. To do this beyond the time when the pinch has reached its quasi-equilibrium point, the value of $\kappa$ is kept fixed at its quasi-equilibrium value and $\mathrm{d} \kappa / \mathrm{d} \tau$ and $\mathrm{d}^{2} \kappa / \mathrm{d} \tau^{2}$ are taken as 0 . This integration shows that in both the examples considered above the magnitude of the voltages on the capacitors and their currents remain within reasonable limits. In the capacitor-Marx combination of the first example $I_{1}$ reaches a second peak of 0.9 of $V_{1} /\left(L_{1} / C_{1}\right)^{1 / 2}$ at $\tau=3.35$ and the voltage on $C_{1}$ reaches a value of $-0.82 V_{1}$ at $\tau=4.0$. For the capacitor-capacitor combination of the second example, because of the greater initial charge on $C_{2}$, the voltage on $C_{1}$ does reach a reverse peak value of $-1.02 V_{1}$ at $\tau=6.1$. In an actual experiment it may be expected that stray resistance and plasma resistive effects will significantly damp these swings. These values do not represent impractical values for the safe operation of the capacitors.

\section{Discussion and conclusions}

The use of a snow-plough equation of motion would by itself produce a non-physical final radius of zero. In the present model the snow-plough trajectory is terminated by the use of an energy balance criterion thus giving a proper non-zero radius ratio. This procedure has certain limitations. For example if a constant current is instantaneously imposed on the pinch the initial condition would be $\tau=0, \iota=1$. It is not possible to start the integration of the snow-plough equation of motion (10) with this condition. This means that the trajectory of the compression cannot be followed even though the final radius ratio may be obtained simply by integrating equation (2). This particular situation could be improved by replacing the structureless snow-plough equation of motion with a model with structure such as the slug model (Potter 1978). It should also be stressed that the model discussed here applies only to fast (supersonic) compressions.

This current-stepping technique has been shown to be able to significantly reduce the plasma pinch ratio. From the results obtained it is evident that further enhancement of the pinch compression may be attained by using multiple steps added to the primary pinching current. It is doubtful though that radius ratios of the order of even 0.01 may be achieved by this technique with practical current generators whilst maintaining the assumption of a lossless plasma. However the pinch compression may be sufficiently enhanced for this technique to he of importance in the design of high intensity soft $x$-ray sources using argon or xenon plasmas.

Also, provided that the pinch is operated with the correct line density to be in the finite Larmor radius stabilised regime (Haines 1982) the current-stepping technique may be useful as a prelude to a final process of compression due to radiation cooling leading to the desired small radius ratios. In terms of the energy balance theory, radiation cooling will lead to a reduced slope in the $F_{R}$ curve in figure $2(b)$. Linking the $F_{R}$ curve to radiation losses will thus result in a further reduction of the final values of $\kappa_{\mathrm{p}}$. 


\section{Acknowledgments}

The assistance of Jalil bin Ali in part of the computation is acknowledged.

\section{References}

Haines M G 1982 Phys. Scr. T2/2 380-90

Lee S 1981 Bul. Fiz. Malaysia 2240

- 1983a Plasma Phys. 25571

— 1983b J. Appl. Phys. 543603

- 1983c Austr. J. Phys.

— 1983d J. Phys. D: Appl. Phys. 162463

Potter D E 1978 Nucl. Fusion 18813 